\title{
LITERATUR
}

\section{Der Raum der Freiheit, der Sicherheit und des Rechts: Analysen zur Europäischen Justiz- und Innenpolitik}

\author{
Friedemann Kainer*
}

Der Raum der Freiheit, der Sicherheit und des Rechts (RFSR) wurde durch den Amsterdamer Vertrag im Jahre 1999 in das europäische Primärrecht eingeführt. Schon zuvor verfügte die dritte Säule der Europäischen Union über - wenn auch intergouvernementale - Zuständigkeiten in der Justiz- und Innenpolitik. Jedoch erst mit der teilweisen Vergemeinschaftung und der Stärkung der dritten Säule insbesondere durch Einführung des Rahmenbeschlusses kam die Entwicklung in Gang. Nach acht Jahren kann die Union nun auf eine rasante Entwicklung zurückblicken: die Schaffung eines gemeinsamen Asylsystems, zahlreiche einwanderungs- und visarechtliche Regelungen, bedeutende Fortschritte in der justiziellen und polizeilichen Zusammenarbeit. Die Literatur zu Einzelbereichen (beispielsweise der Zusammenarbeit im Zivilverfahren oder dem europäischen Asylrecht) ist kaum überschaubar; Untersuchungen, die über Einzelfragen hinausgehen und die Justizund Innenpolitik als Ganzes untersuchen, sind hingegen weniger zahlreich. Die nachstehend besprochenen Monographien und Sammelbände gehen dem rechts- beziehungsweise politikwissenschaftlichen Gesamtbild des RFSR nach und lohnen daher näherer Betrachtung.

\section{Rechtlicher Rahmen}

Will man sich der europäischen Justiz- und Innenpolitik nähern, so wird man zunächst kaum an dem ebenso umfassenden wie aktuellen Werk von Steve Peers vorbeikommen. Es entwickelt einen ebenso umfassenden wie
Steve Peers: EU Justice and Home Affairs Law, [Series: Oxford European Community Law Library], 2nd edition, Oxford University Press: Oxford 2006, ISBN 978-0-19-929055-0; 588 Seiten, $75 £$.

Peter-Christian Müller-Graff (Hrsg.): Der Raum der Freiheit, der Sicherheit und des Rechts, [Schriftenreihe des Arbeitskreises Europäische Integration e.V., Band 51], Nomos Verlagsgesellschaft: Baden-Baden 2005, ISBN 978-38329-1101-0; 185 Seiten, $36 €$.

Eckhard Pache (Hrsg.): Die Europäische Union Ein Raum der Freiheit, der Sicherheit und des Rechts?, [IUS EUROPAEUM, Band 35], Nomos Verlagsgesellschaft: Baden-Baden 2005, ISBN 978-3-8329-1429-5; 155 Seiten, $34 €$.

Joanna Apap (Hrsg.): Justice and Home Affairs in the EU. Liberty and Security Issues after Enlargement, Edward Elgar: Cheltenham/Northampton 2004, ISBN 978-1-84376-787-9; 360 Seiten, $65 £$.

Jef Huysmans: The Politics of Insecurity. Fear, migration and asylum in the EU, [New International Relations Series], Routledge: London/New York 2006, ISBN 9-78-0415-36125-5; 208 Seiten, $19.99 £$.

Franck Düvell (Hrsg.): Illegal Immigration in Europe. Beyond Control?, Palgrave Macmillan: Basingstoke/New York 2006, ISBN 1-40399741-1; 288 Seiten, $52 £$.

konzisen und systematischen Blick auf das Recht der europäischen Innen- und Justizpolitik. Peers beschränkt sich dabei nicht auf

* Dr. Friedemann Kainer, Institut für deutsches und europäisches Gesellschafts- und Wirtschaftsrecht, Universität Heidelberg. 
Maßnahmen der dritten Säule beziehungsweise der Artikel 61 ff. EG-Vertrag, sondern erörtert auch Regelungen des sonstigen $\mathrm{Ge}$ meinschaftsrechts, soweit sie an betroffene Sachfragen anschließen. Seine Darstellung steht dabei unter vier Grundfragen: das Verhältnis zwischen europäischer Justiz- und Innenpolitik zu den europäischen und internationalen Grundrechten, das Verhältnis zwischen Maßnahmen erster und dritter Säule, die Reichweite der Kompetenzen und schließlich die territoriale Anwendbarkeit der europäischen Justiz- und Innenpolitik. Dieses Schema wird zunächst in einem ausführlichen Kapitel zum institutionellen Rahmen entwickelt, in dem Peers unter anderem auf die Entwicklungsstadien und den geltenden rechtlichen, grundrechtlichen und territorialen Rahmen eingeht. Durch die Verknüpfung seiner Grundfragen mit den Einzelproblemen werden zahlreiche Querbeziehungen erkenntnisfördernd offengelegt. Bei allem werden die aktuellen Entwicklungen in Politik, Rechtsakten und Rechtsprechung erhellend und nahezu erschöpfend dargestellt und analysiert, wobei vielfach die britische Sicht hervorschimmert was das Buch für den kontinentaleuropäischen Leser noch bereichernder macht. Alles in allem ein analytisch aufgebautes, systematisch überzeugendes und lesenswertes Werk.

Mit zahlreichen Einzelaspekten beschäftigen sich zwei Tagungsbände von Peter-Christian Müller-Graff und Eckhard Pache. Dem von Müller-Graff herausgegebenen Werk lag eine Tagung im Mai 2003 in Heidelberg zugrunde, die sich mit den primärrechtlichen Grundlagen, der politischen Konzeption und den Inhalten des Raums der Freiheit, der Sicherheit und des Rechts beschäftigte. Im ersten Teil führen zunächst Müller-Graff in die rechtliche und Jörg Monar in die politische Konzeption des neuen Politikbereichs ein. Das Nebeneinander beider Beiträge ist interessant, weil es ein Spannungsverhältnis zwischen der binnenmarktrechtlichen Fundierung einerseits und den politisch erhofften Inhalten des letztlich unkonturierten Zieldreiecks (Freiheit, Sicherheit, Recht) andererseits erkennen lässt.
Charles Elsen gibt im Anschluss einen Einblick zur Entstehung und Zukunft des Raums der Freiheit, der Sicherheit und des Rechts aus der Sicht des Rates. In den folgenden Teilen werden die primärrechtlichen Grundlagen und die Entwicklung der einzelnen Politikbereiche dargestellt und analysiert, wobei der Beitrag von Thorsten Müller querschnittsartig die Außenpolitik des Raums der Freiheit, der Sicherheit und des Rechts beleuchtet. Im Rahmen der polizeilichen Zusammenarbeit entwerfen die Beiträge von Herrmann von Langsdorff und Harald Felgenhauer eine höchst interessante Innensicht von Eurojust und Europol. Auch die Untersuchung der interkulturellen Zusammenarbeit nationaler Polizeikräfte (Rainer Pitschas) und ein Landesbericht zur polizeilichen und strafjustiziellen Zusammenarbeit in Deutschland speisen empirische Daten in die Diskussion ein, an denen es vielfach mangelt. Insgesamt ist der Band, dem allenfalls eine Analyse der Rechtsentwicklung in der dritten Säule fehlen mag, wegen des grundlegenden Untersuchungsfokus der einzelnen Beiträge beziehungsweise ihrer Innensichten in jeder Hinsicht lesenswert.

Der Band von Pache ging aus einer Tagung in Würzburg im Juni 2004 hervor und analysiert in sieben Beiträgen aktuelle Grundfragen des Raums der Freiheit, der Sicherheit und des Rechts auf der Basis des projektierten Verfassungsvertrages. Die Grundlage hierzu legt nach einer Standort- und Begriffsbestimmung von Pache - Matthias Ruffert, der den neuen Vertrag in den Politikbereichen Justiz und Inneres in einen verfassungstheoretischen Rahmen einordnet und in Kompetenzen, Verfahren und gerichtlicher Kontrolle knapp darstellt. Die folgenden Aufsätze beschäftigen sich mit Einzelthemen. Zwei Beiträge widmen sich der polizei- und strafrechtlichen $\mathrm{Zu}$ sammenarbeit, wobei sich vor allem Manfred Baldus deutlich kritisch mit der Europäisierung des Polizeirechts auseinandersetzt und Konsequenzen für das tradierte Konzept moderner Staatlichkeit zieht: Neuakzentuierungen im Hinblick auf die Bedeutung von Grenzen für die Ausübung von Hoheitsgewalt und 
die Zuordnung des Gewaltmonopols. Mit der Vorstellung des Alternativentwurfs zur Regelung der europäischen Strafverfolgung setzt auch Bernd Schünemann einen eher kritischen Akzent. Dagegen bewertet der Beitrag von Eckhart von Bubnoff, der sich mit der Rechtsetzung im Raum der Freiheit, der Sicherheit und des Rechts - eher aus strafrechtlicher Perspektive - beschäftigt, die Entwicklung eher positiv. Weitere Themen des Bandes sind das europäische Zuwanderungsrecht (Albrecht Weber) und die Harmonisierung des europäischen Umweltstrafrechts (Jörg Eisele). Auch dieser Band stellt gerade in seiner Meinungsvielfalt einen wertvollen Beitrag zur Entwicklung der europäischen Justiz- und Innenpolitik dar.

Der RFSR vor dem Hintergrund der Erweiterung

Speziell mit der Erweiterung der Europäischen Union beschäftigen sich die zahlreichen Beiträge des von Joanna Apap herausgegebenen Bandes. Die mit der Erweiterung verbundenen Fragen im Rahmen der europäischen Justiz- und Innenpolitik sind in der Tat höchst untersuchungswürdig. Neue Probleme ergeben sich aus der Notwendigkeit der Umsetzung des Acquis und tatsächlichen Schwierigkeiten bei der Sicherung der Außengrenzen, der größeren Unterschiedlichkeit der Rechtssysteme und -traditionen, neuen immigrationspolitischen Problemen sowie größeren Schwierigkeiten einiger neuer Mitgliedstaaten bei der gemeinschaftlichen Ausübung von Hoheitsrechten im Rahmen souveränitätssensibler Bereiche der dritten Säule. Der Band ist in drei Teile aufgeteilt, deren erster in die Grundlagen der europäischen Justiz- und Innenpolitik nach der Erweiterung einführt. Sarah Ludford arbeitet grundlegende Strukturmerkmale für den politischen Rahmen heraus, Jörg Monar analysiert politische und rechtliche Wege zur Lösung der durch die Erweiterung induzierten Hemmnisse für die weitere Entwicklung. Ein längerer Beitrag von Paul de Hert beschäftigt sich mit der Aufteilung der Kompetenzen zwischen natio- naler und europäischer Ebene und zwischen erstem und drittem Pfeiler der Union. De Hert spricht sich dabei im Hinblick auf das Demokratiedefizit und den unzureichenden Grundrechtsschutz sehr dezidiert gegen weitere $\mathrm{Zu}$ ständigkeiten in der dritten Säule aus. Im zweiten Teil folgen Beiträge, die sich mit innenpolitischen Szenarien vor allem in der Kriminalitätsbekämpfung und -prävention befassen. Die behandelten Themen sind bunt, vom Grenzschutz im erweiterten Europa über das Grundrecht auf gute Verwaltung, den Wiederaufbau der Justizsysteme in den Staaten des Stabilitätspakts für Südosteuropa bis hin zu OLAF und seiner Kooperation mit Behörden der neuen Mitgliedstaaten. Ähnlich gestaltet sich auch der dritte Teil, der Themen um die zukünftige Einwanderungs- und Asylpolitik in Europa behandelt. Die Einzelbeiträge sind höchst interessant, etwa Elspeth Guilds Analyse möglicher Modelle für eine europäische Regelung der Arbeitsmigration oder die Untersuchung der gemeinsamen Asylpolitik in Europa von Johannes van der Klaauw, der aus der Perspektive der UNHCR für ein offenes Asylsystem plädiert. Am Ende finden sich fünf konkrete Empfehlungen, die von Apap als Ergebnisse der Beiträge formuliert werden, und die eine sehr schöne Klammer um die vorausgehenden (inhaltlich nicht immer sichtbar einem roten Faden folgenden) Aufsätze bilden.

Politische und soziologische Grundlagen der europäischen Zuwanderungspolitiken

Eine Feststellung von Apap, dass Zuwanderungspolitiken oft zu sehr am Maßstab tatsächlicher oder auch nur imaginärer Sicherheitsbedenken ausgerichtet werden, ist Gegenstand eines neueren Beitrags von Jef Huysmans. Aus einem politikwissenschaftlichen und soziologischen Blickwickel beschäftigt sich Huysmans mit dem Verhältnis zwischen Sicherheit und Migrations- und Asylpolitik. Ausgangspunkt der theoretisch eindrucksvollen und auf Foucault basierenden Argumentation ist eben jene Feststellung, dass in der EU Migrations- und Asylpolitik zu 
einseitig sicherheitspolitisch verstanden wird. Konstruiert man Migrationspolitik unter dem Gesichtspunkt allgegenwärtiger Gefährdungen der Sicherheit (durch Kriminalität, Lohndumping etc.), so führt dies dazu, dass sich die betroffenen Politiken entsprechend definieren; die Konstruktion spurt die Inhalte vor. Migration und Asyl verlieren weitgehend ihre individual- und grundrechtlichen Fundamente und werden zu einer „Politik der Unsicherheit“. Diese Ausgangslage führt den Verfasser zu einer Reihe von weiterführenden Fragestellungen, die in acht Kapiteln entwickelt werden. Am Anfang untersucht Huysmans die Grundlagen des Sicherheitsbegriffs, der nach Ende des Kalten Krieges erhebliche Modifikationen erhalten hat: Nachdem früher Konflikte zwischen Staaten im Mittelpunkt standen, werden jetzt Gefährdungen etwa aus Zuwanderungsprozessen sicherheitspolitisch relevant. Huysmans erörtert die Eingangstore von Sicherheitstopoi gerade in der Migrationspolitik und führt in die spezifische Situation der Europäischen Union ein. Seine Argumentation geht dahin, dass das Vordringen von Sicherheitspolitik ein multikausaler, aber ,gewollter' Prozess ist (political choice), der sich nicht auf die Terroranschläge des Jahres 2001 alleine zurückführen lässt. Huysmans streitet für eine Neujustierung des Verhältnisses zwischen Sicherheit und Freiheit, insgesamt für eine Entkoppelung der Zuwanderungspolitik vom Sicherheitsdenken, und entwickelt hierzu die theoretischen Grundlagen. Bei allem findet der Leser ein dichtes, gedanken- und themenreiches Werk auf höchstem theoretischem Niveau vor, das allenfalls mitunter stärker empirisch fundiert sein könnte. Es nimmt eine der zentralen politischen Fragen der Gegenwart auf und dürfte über die Migrationspolitik hinaus gute Aufnahme in der Wissenschaft finden.

Auf die Hintergründe von illegaler Einwanderung in Europa geht das von Franck Düvell herausgegebene und überwiegend von ihm verfasste Buch ein. Es stellt die Frage nach den Gründen für die (abwehrende) Immigrationspolitik in der Europäischen Union und ih- ren Folgen. In vier Abschnitte aufgeteilt, erhellt das Werk zunächst kritisch die geschichtliche Entwicklung des Begriffs der illegalen Einwanderung, ihre ökonomischen Gründe und (positiven!) Wirkungen sowie die bisherige Forschung zum Thema. In Fallstudien geht der zweite Teil auf individuelle Schicksale von Einwanderern in Großbritannien, Deutschland, Italien und Griechenland ein. Die Ergebnisse dieser Fallstudien enthüllen vor allem die Dynamik der Einwanderer und eine erstaunliche Rationalität ihrer Entscheidungen. Einwanderer verhalten sich nutzenmaximierend gerade so, wie es in einem Lehrbuch zur Theorie der rationalen Entscheidung beschrieben ist. Interessant sind vor allem auch die unterschiedlichen Einwanderungsregime in den untersuchten Staaten, die in Details aus Sicht der Einwanderer selbst zum Ausdruck kommen. In der Analyse eröffnet Düvell interessante Perspektiven; so zeigt er, wie verschiedene soziale und wirtschaftliche Systeme unterschiedliche Charakteristika von Migranten anziehen. Spannend ist auch die Analyse der Gerechtigkeitsvorstellungen von Immigranten: den wenigsten geht es darum, Gebrauch von den sozialen Systemen der Aufnahmestaaten zu machen, in der Regel sind sie stolz, wirtschaftlich zum Erfolg ihrer neuen oder vorübergehenden Heimat beizutragen. Auch deswegen fühlen sich Immigranten, die kaum je - so Düvell - einem einheimischen Arbeitnehmer direkt Konkurrenz machen, moralisch im Recht. Im letzten Kapitel setzt sich Düvell mit verschiedenen Politikansätzen zur Lösung des Migrationsproblems auseinander. Migration ist für ihn einerseits ein anthropologisches Bedürfnis, andererseits Zeichen eines sozialen Konflikts, dessen Verhinderung allgemeinen Gerechtigkeitsvorstellungen widerspreche. Da Migration wegen der Marktmechanismen und der Subjektivität menschlichen Handelns nicht verhindert werden könne, sondern zuwandernde Arbeitnehmer lediglich in die Schattenwirtschaft dränge, sei das derzeitige Modell der meisten EU-Staaten und auch die EU-Politik letztlich gescheitert. Die empirischen Befunde untermauern diese These eindrucksvoll. 
Düvells Ansatz für eine gerechte Migrationspolitik betont den liberalen Grundsatz der Gleichheit aller Menschen und ihrer Autonomie. Er plädiert für Einbeziehung und Integration statt Ausschluss und Illegalisierung von Migranten. Die Erweiterung der Europäischen Union dient ihm als Beispiel. Man mag Düvell gerne beipflichten, dass Migration viele positive Effekte hervorbringt; jedoch fokussiert sich die Untersuchung leider überwiegend auf europäische Einwanderer, ohne die zum Teil anderen Fragestellungen und Gegebenheiten bei afrikanischen und asiatischen Flüchtlingen hinreichend zu diskutieren. Vor allem aber blendet Düvell bei seiner am Ende ein wenig knappen Argumentation Schwierigkeiten der Integration von Flüchtlingen und Probleme der Herkunftsstaaten (beispielsweise ,brain drain') aus und bleibt damit vielleicht etwas einseitig. Gleichwohl ist das Werk gerade wegen seiner individuellen Blickrichtung ein bedeutsamer Beitrag zur immigrationspolitischen Debatte in Europa.

\section{Verbleibende Forschungsfelder}

Die hier besprochenen, für den Politikbereich nur beispielhaften Monographien und Sam- melbände lassen eine immer stärkere wissenschaftliche Durchdringung der europäischen Innen- und Justizpolitik erkennen. Die steigende Zahl sowohl an rechtlichen wie auch politikwissenschaftlichen Publikationen reflektiert das in einem größeren Zusammenhang geradehin Revolutionäre der gemeinschaftlichen Ausübung von Politik in Kernbereichen staatlicher Souveränität. Sie reflektiert aber auch eine verfassungsrechtliche Verflechtung in Europa, die in dynamischer Entwicklung befindlich und daher in vielen Einzelbereichen selbst noch unzureichend erforscht ist. Die Spannungsverhältnisse, die Anlass für weitere Arbeiten geben, sind vielfältig: Sie bewegen sich zwischen nationaler und europäischer Kompetenz (Zuständigkeitsproblem), zwischen supranationaler und intergouvernementaler Ausgestaltung (Abgrenzungsproblem), zwischen demokratisch-parlamentarischer oder völkerrechtlicher Legitimation auf Basis der Staatengleichheit (Legitimationsproblem), zwischen Freiheit und Sicherheit (Grundrechtsproblem), zwischen staatlichen und individuellen Interessen (Migrationsproblem). Über einen Mangel an offenen Fragen ist nicht zu klagen.

\section{Regionale Vision des Balkans}

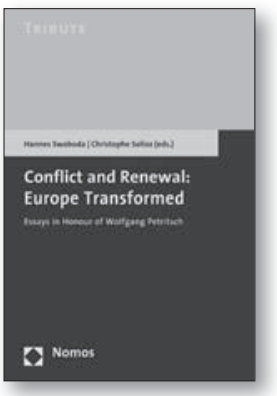

\section{Conflict and Renewal: Europe Transformed}

Essays in Honour of Wolfgang Petritsch

Herausgegeben von Hannes Swoboda und Christophe Solioz

2007, 407 S., brosch., 79,- $€$, ISBN 978-3-8329-2843-8

Hochrangige internationale Persönlichkeiten aus Politik, Wissenschaft und Gesellschaft haben anlässlich des 60 . Geburtstags von Wolfgang Petritsch Beiträge für den Band geschrieben.

Bitte bestellen Sie bei Ihrer Buchhandlung oder bei Nomos | Telefon 07221/2104-37 | Fax -43 www.nomos.de | sabine.horn@nomos.de

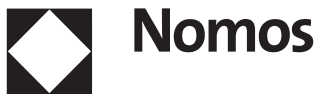

